\title{
Composition tuning of Ru-based phosphide for enhanced propane selective dehydrogenation
}

Rui Ma ${ }^{1,2}$, Tianxing Yang ${ }^{1}$, Junxian $\mathrm{Gao}^{2}$, Jiajing Kou ${ }^{2,3}$, Johnny Zhu Chen ${ }^{2}$, Yufei He ${ }^{1, *}$, Jeffrey TMiller, ${ }^{2, *}$ and Dianqing $\mathrm{Li}^{1, *}$

${ }^{1}$ State Key Laboratory of Chemical Engineering, Beijing University of Chemical Technology, Beijing, 100029 (P.R. China)

${ }^{2}$ Davidson School of Chemical Engineering, Purdue University, 480 Stadium Mall Drive, West Lafayette, Indiana 47907, United States

${ }^{3}$ State Key Laboratory of Multiphase Flow in Power Engineering (SKLMF), Xi'an Jiaotong University, 28 Xianning West Road, Xi'an, 710049, Shaanxi, China
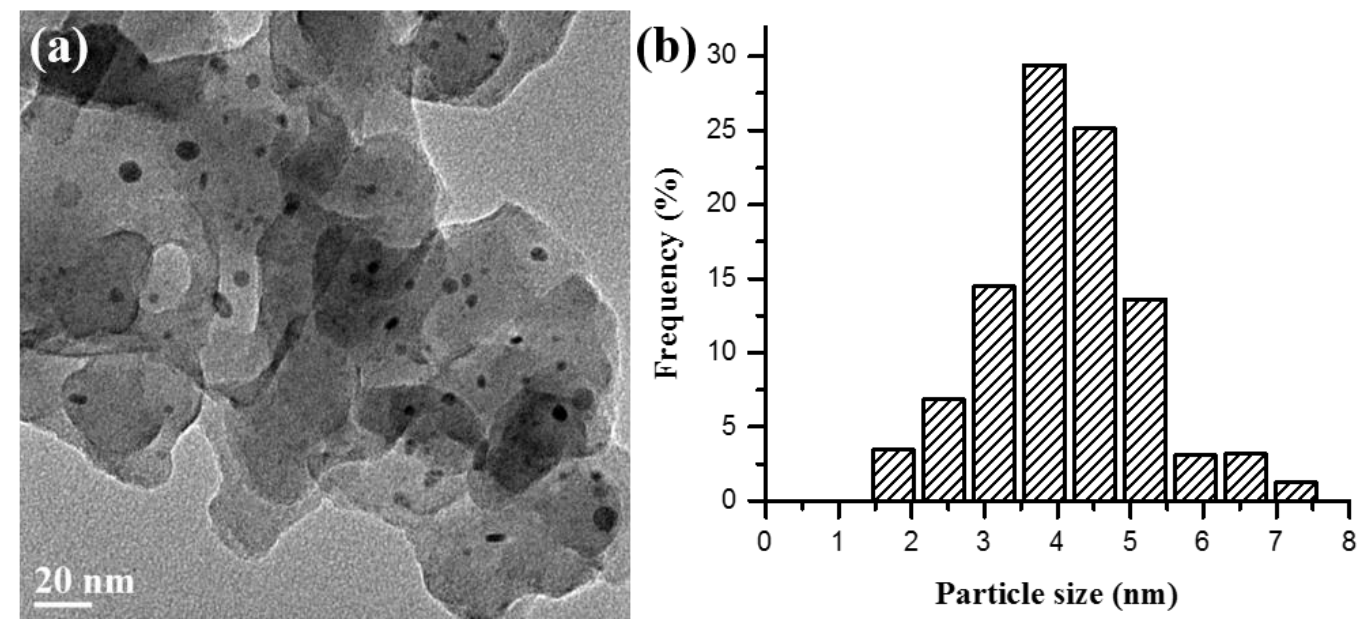

Figure S1. Transmission electron microscopy micrographs (a) and histograms of metal particle size distribution (b) for $\mathrm{Ru} / \mathrm{SiO}_{2}$ sample. 


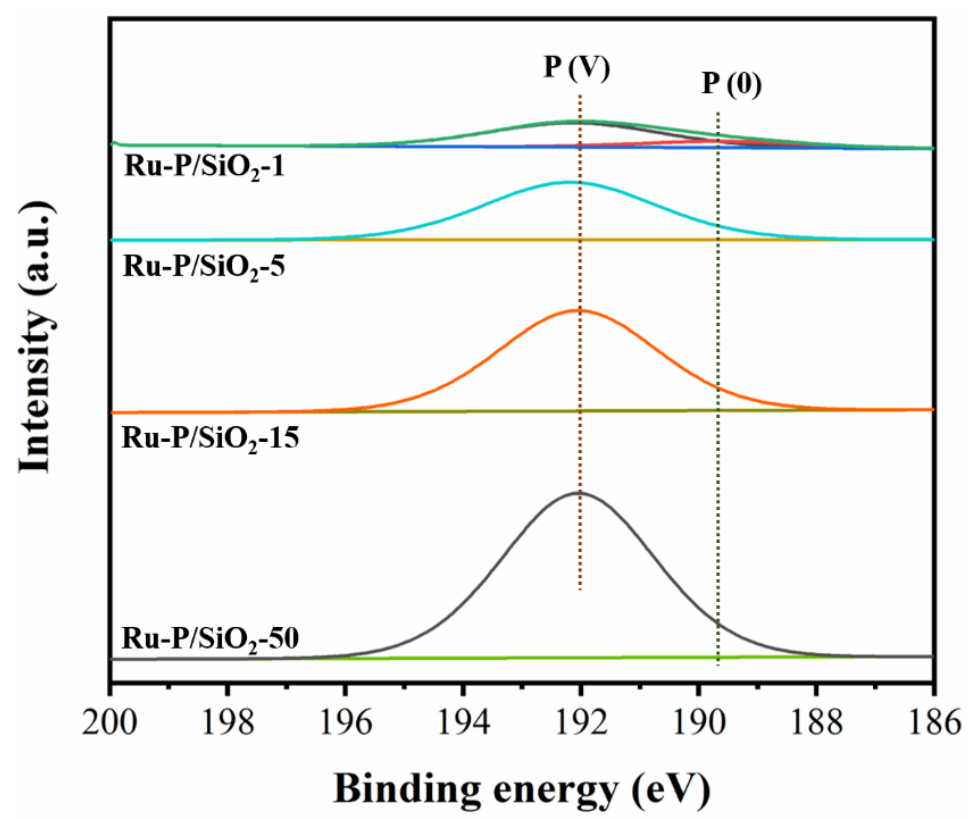

Figure S2. XPS spectrum of P 2s region for series Ru-P samples with different P/Ru ratios.
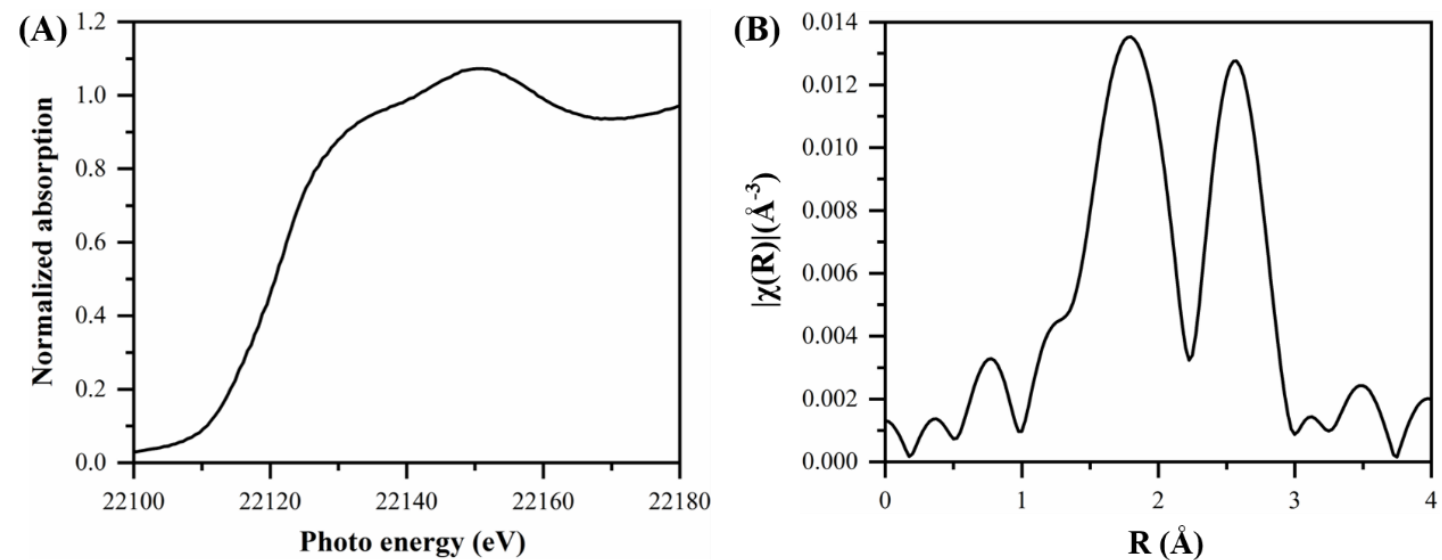

Figure S3. Ru K-edge (A) XANES spectra and (B) magnitude of the Fourier transform of the EXAFS of $\mathrm{Ru}-\mathrm{P} / \mathrm{SiO}_{2}-5$. 

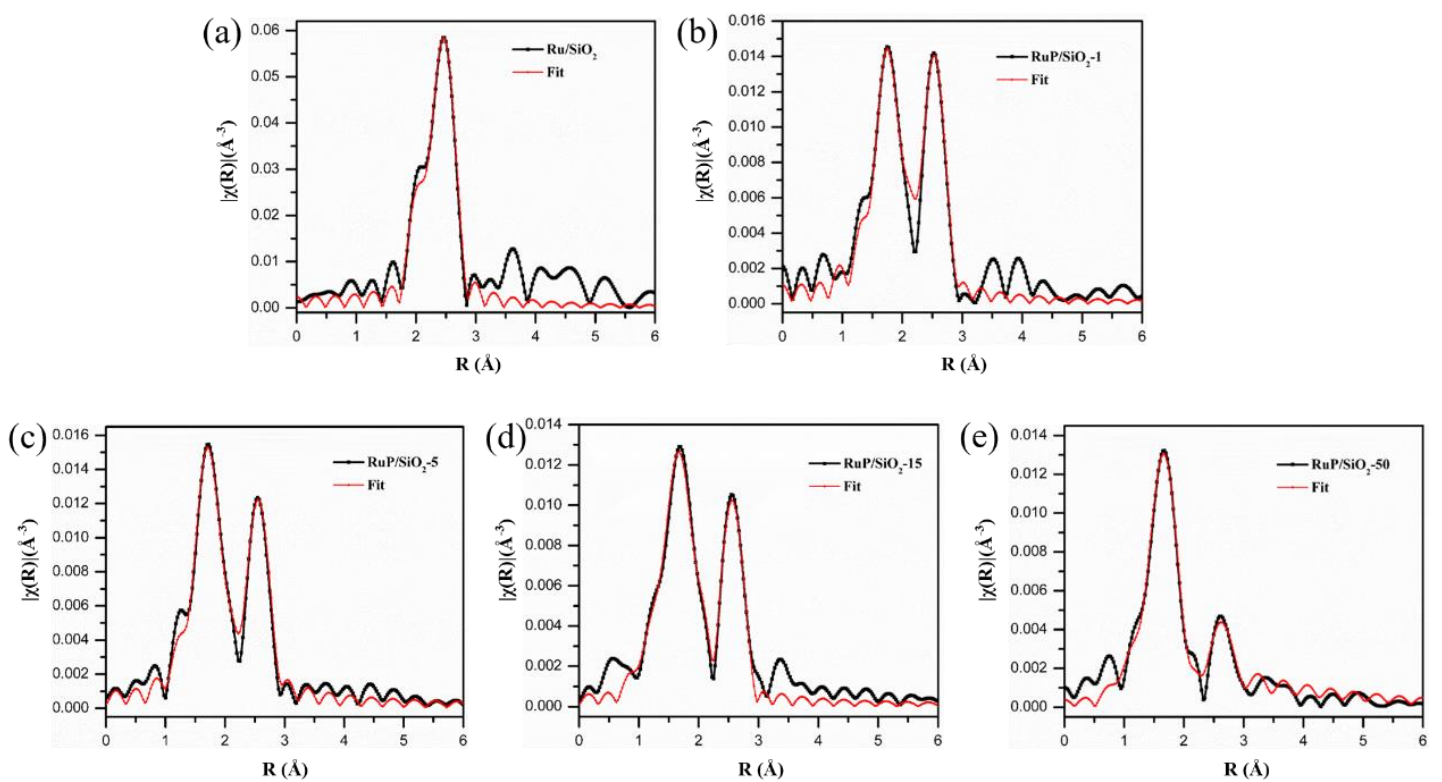

Figure S4. Fourier transform magnitudes of $\mathrm{k}^{2}$-weighted EXAFS data and theoretical fits of (a) $\mathrm{Ru} / \mathrm{SiO}_{2}$, (b) $\mathrm{Ru}-\mathrm{P} / \mathrm{SiO}_{2}-1$, (c) $\mathrm{Ru}-\mathrm{P} / \mathrm{SiO}_{2}-5$, (d) $\mathrm{Ru}-\mathrm{P} / \mathrm{SiO}_{2}-15$, and (e) $\mathrm{Ru}-\mathrm{P} / \mathrm{SiO}_{2}-50$.
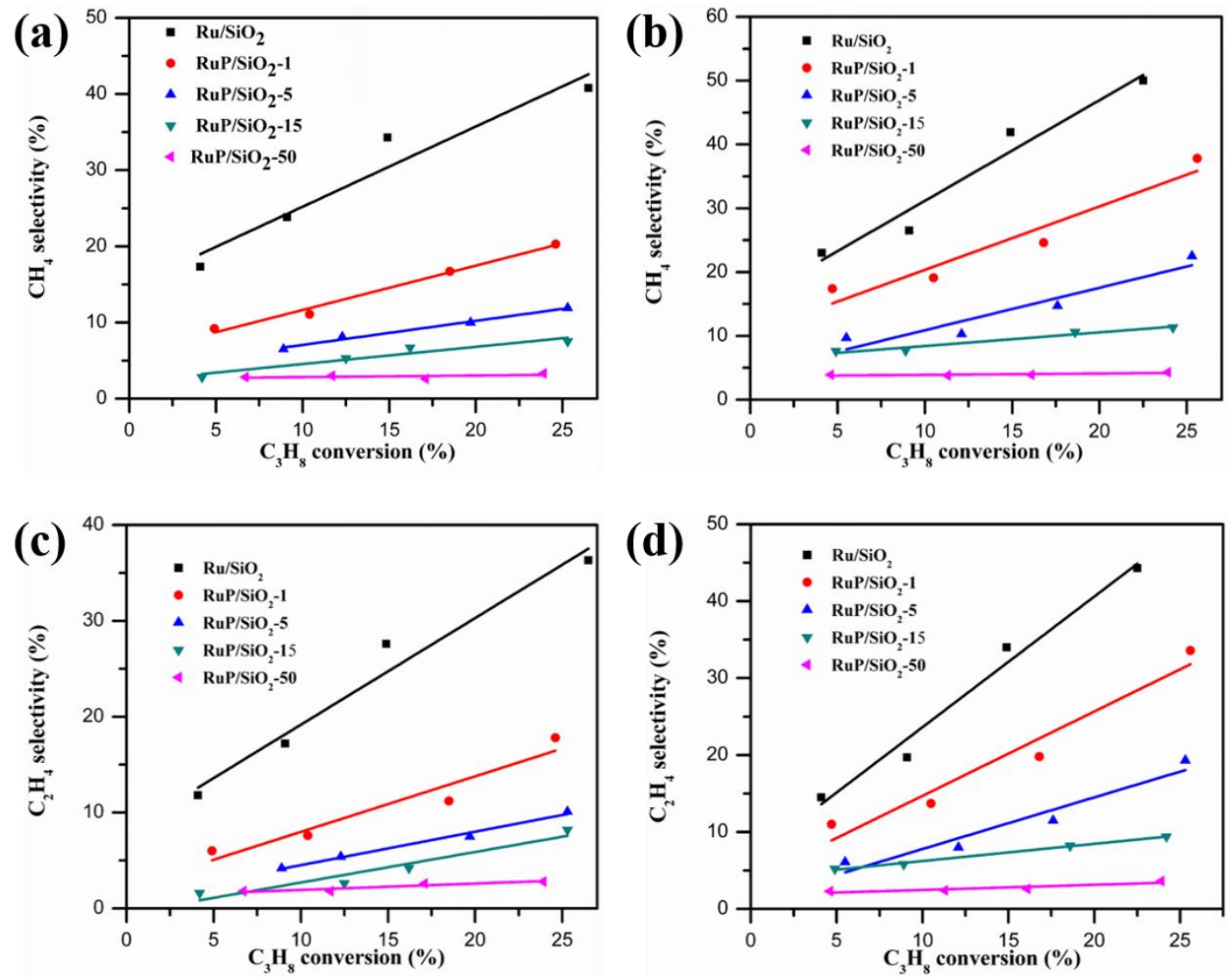

Figure S5. Initial methane and ethylene selectivity versus propane conversion for Ru-P catalysts in propane dehydrogenation reaction at $(\mathrm{a}, \mathrm{c}) 550{ }^{\circ} \mathrm{C}$ and $(\mathrm{b}, \mathrm{d}) 600{ }^{\circ} \mathrm{C}$ compared with that for 
monometallic Ru catalyst.
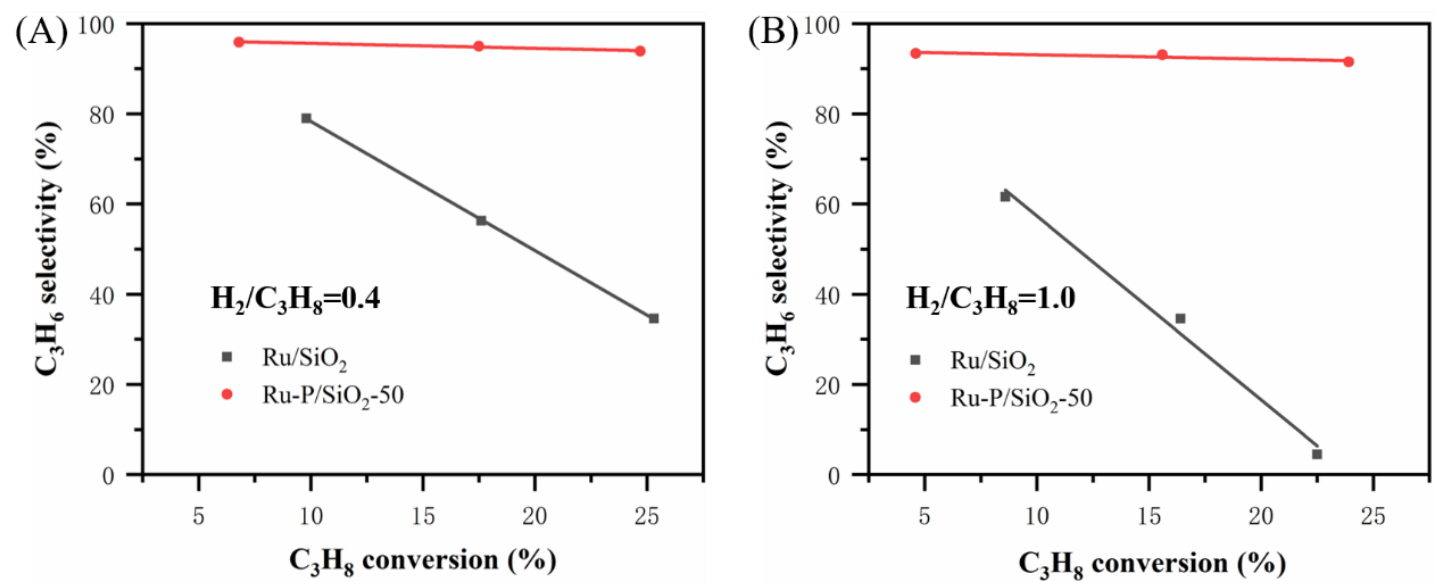

Figure S6. Conversion vs selectivity at $600{ }^{\circ} \mathrm{C}$ and atmospheric pressure with 0.4 (A) and 1.0 (B) $\mathrm{H}_{2} /$ propane ratios.

Table S1. Catalytic conditions of $\mathrm{C}_{3} \mathrm{H}_{8}$ dehydrogenation at $\sim 25 \%$ propane conversion and corresponding reaction rate. ${ }^{a}$

\begin{tabular}{cccc}
\hline Catalyst & $\begin{array}{c}\text { Catalyst loading } \\
(\mathrm{g})\end{array}$ & $\begin{array}{c}\mathrm{C}_{3} \mathrm{H}_{8} \text { conversion } \\
(\%)\end{array}$ & $\begin{array}{c}\text { Rate } \\
\left(\mathrm{mol}_{\mathrm{C} 3} / \mathrm{mol}_{\mathrm{P} t \mathrm{Ru}} \mathrm{s}^{-1}\right)\end{array}$ \\
\hline $\mathrm{Pt} / \mathrm{SiO}_{2}$ & 0.10 & 24.9 & 0.0139 \\
$\mathrm{Ru} / \mathrm{SiO}_{2}$ & 0.30 & 24.7 & 0.0024 \\
$\mathrm{Ru}-\mathrm{P} / \mathrm{SiO}_{2}-1$ & 0.40 & 25.6 & 0.0019 \\
$\mathrm{Ru}-\mathrm{P} / \mathrm{SiO}_{2}-5$ & 0.40 & 25.3 & 0.0018 \\
$\mathrm{Ru}-\mathrm{P} / \mathrm{SiO}_{2}-15$ & 0.40 & 24.8 & 0.0018 \\
$\mathrm{Ru}-\mathrm{P} / \mathrm{SiO}_{2}-50$ & 0.40 & 25.8 & 0.0019 \\
\hline
\end{tabular}

${ }^{a}$ performing at $600{ }^{\circ} \mathrm{C}$ with $2.5 \% \mathrm{C}_{3} \mathrm{H}_{8}$ and $2.5 \% \mathrm{H}_{2}$ at a total flow of $100 \mathrm{~mL} / \mathrm{min}$. 


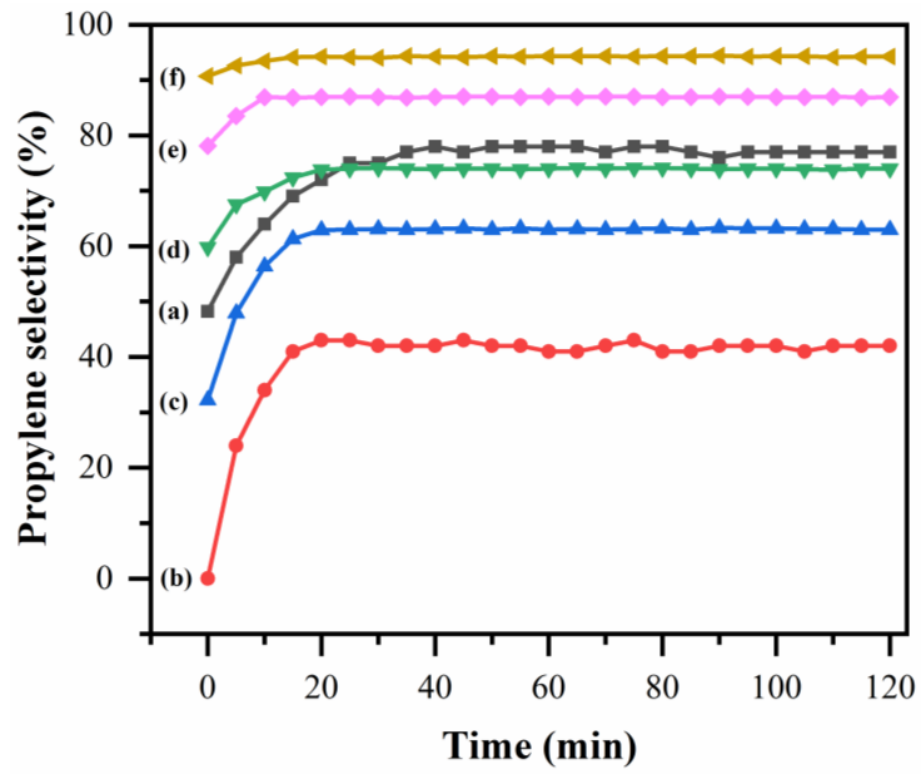

Figure S7. The propylene selectivity during the propane dehydrogenation reactions over (a) $\mathrm{Pt} / \mathrm{SiO}_{2}$, (b) $\mathrm{Ru} / \mathrm{SiO}_{2}$, (c) Ru-P/SiO${ }_{2}-1$, (d) Ru-P/SiO $2-5$, (e) Ru-P/SiO $2-15$, and (f) $\mathrm{Ru}-\mathrm{P} / \mathrm{SiO}_{2}-50$ catalysts (T $=600{ }^{\circ} \mathrm{C}$, atmospheric pressure, $\mathrm{C}_{3} \mathrm{H}_{8} / \mathrm{H}_{2}=1 / 1$, with balance $\mathrm{N}_{2}$ for a total flow rate of $100 \mathrm{~mL} / \mathrm{min}$ ).

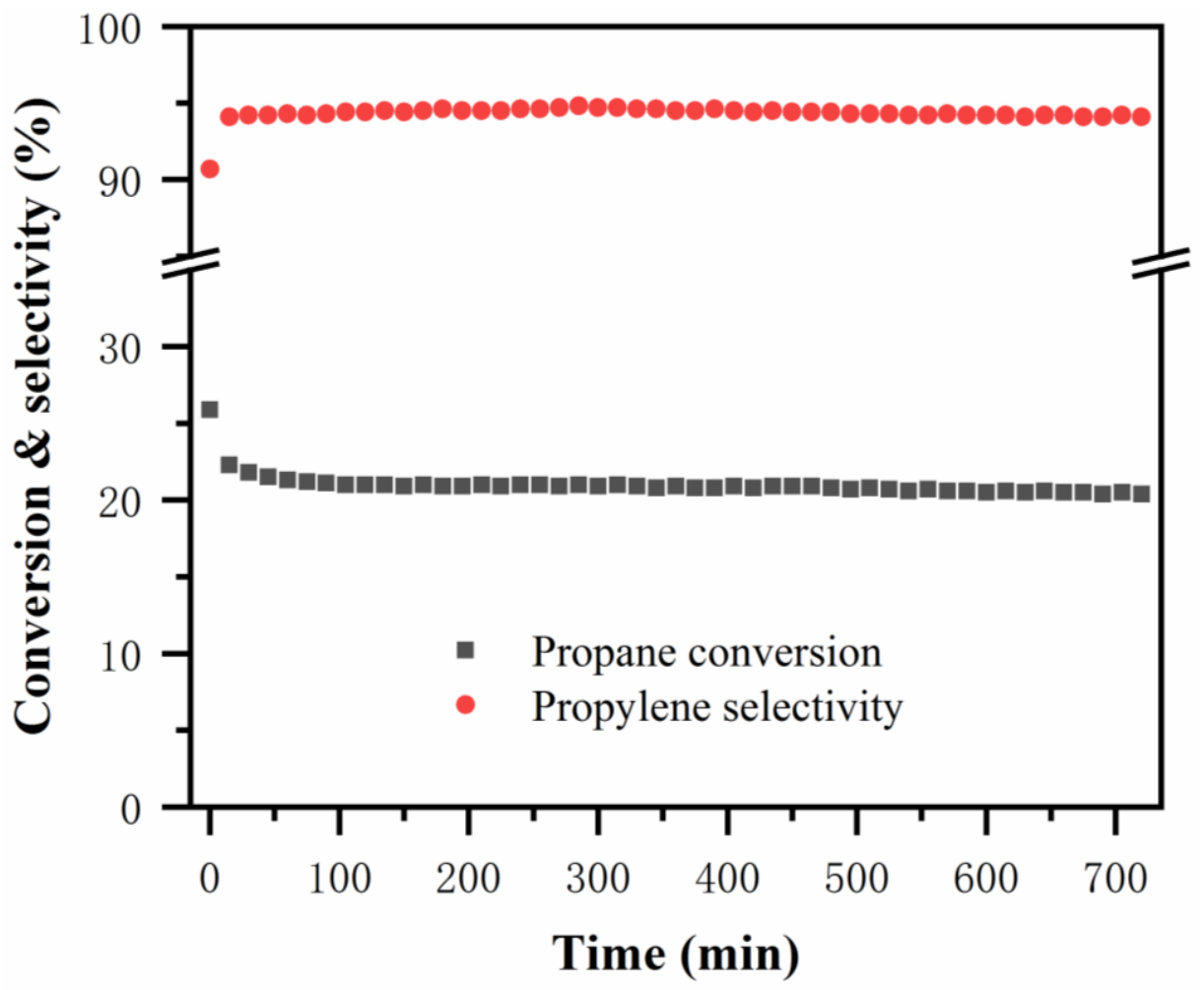

Figure S8. Propane conversion and propylene selectivity during $12 \mathrm{~h}$ over $\mathrm{Ru}-\mathrm{P} / \mathrm{SiO}_{2}-50$ catalyst (T $=600{ }^{\circ} \mathrm{C}$, atmospheric pressure, $\mathrm{C}_{3} \mathrm{H}_{8} / \mathrm{H}_{2}=1 / 1$, with balance $\mathrm{N}_{2}$ for a total flow rate of $100 \mathrm{~mL} / \mathrm{min}$ ). 